\title{
Tracking suspected heroin overdoses in CDC's National Syndromic Surveillance Program
}

\author{
Alana M. Vivolo-Kantor ${ }^{* 1}$, R. Matthew Gladden', Aaron Kite-Powell ${ }^{2}$, Michael Coletta ${ }^{2}$ \\ and Grant Baldwin'
}

${ }^{1}$ Division of Unintentional Injury Prevention, Centers for Disease Control and Prevention, Atlanta, GA, USA; ${ }^{2}$ Division of Health

Informatics and Surveillance, Centers for Disease Control and Prevention, Atlanta, GA, USA

\section{Objective}

This paper analyzes emergency department syndromic data in the Centers for Disease Control and Prevention's (CDC) National Syndromic Surveillance Program's (NSSP) BioSense Platform to understand trends in suspected heroin overdose.

\section{Introduction}

Overdose deaths involving opioids (i.e., opioid pain relievers and illicit opioids such as heroin) accounted for at least $63 \%(\mathrm{~N}=33,091)$ of overdose deaths in 2015. Overdose deaths related to illicit opioids, heroin and illicitly-manufactured fentanyl, have rapidly increased since 2010. For instance, heroin overdose deaths quadrupled from 3,036 in 2010 to 12,989 in 2015 . Unfortunately, timely response to emerging trends is inhibited by time lags for national data on both overdose mortality via vital statistics (8-12 months) and morbidity via hospital discharge data (over 2 years). Emergency department (ED) syndromic data can be leveraged to respond more quickly to emerging drug overdose trends as well as identify drug overdose outbreaks. CDC's NSSP BioSense Platform collects near real-time ED data on approximately two-thirds of ED visits in the US. NSSP's data analysis and visualization tool, Electronic Surveillance System for the Notification of Community-based Epidemics (ESSENCE), allows for tailored syndrome queries and can monitor ED visits related to heroin overdose at the local, state, regional, and national levels quicker than hospital discharge data.

\section{Methods}

We analyzed ED syndromic data using ESSENCE to detect monthly and annual trends in suspected unintentional or undetermined heroin overdose by sex and region for those 11 years and older. An ED visit was categorized as a suspected heroin overdose if it met several criteria, including heroin overdose ICD-9-CM and ICD-10-CM codes (i.e., 965.01 and E850.0; T40.1X1A, T40.1X4A) and chief complaint text associated with a heroin overdose (e.g., "heroin overdose"). Using computer code developed specifically for ESSENCE based on our case definition, we queried data from 9 of the 10 HHS regions from July 2016-July 2017. One region was excluded due to large changes in data submitted during the time period. We conducted trend analyses using the proportion of suspected heroin overdoses by total ED visits for a given month with all sexes and regions combined and then stratified by sex and region. To determine significant linear changes in monthly and annual trends, we used the National Cancer Institute's Joinpoint Regression Program.

\section{Results}

From July 2016-July 2017, over 72 million total ED visits were captured from all sites and jurisdictions submitting data to NSSP. After applying our case definition to these records, 53,786 visits were from a suspected heroin overdose, which accounted for approximately 7.5 heroin overdose visits per 10,000 total $\mathrm{ED}$ visits during that timeframe. The rate of suspected heroin overdose visits to total ED visits was highest in June 2017 (8.7 per 10,000) and lowest in August 2016 (6.6 per 10,000 visits). Males accounted for a larger rates of visits over all months (range $=10.7$ to 14.2 per 10,000 visits) than females (range $=3.8$ to 4.7 per 10,000 visits). Overall, compared to July 2016, suspected heroin overdose ED visits from July 2016 were significantly higher for all sexes and US regions combined $(\beta=.010, p=.036)$. Significant increases were also demonstrated over time for males $(\beta=.009, p=.044)$ and the Northeast $(\beta=.012$, $p=.025)$. No other significant increases or decreases were detected by demographics or on a monthly basis.

\section{Conclusions}

Emergency department visits related to heroin overdose increased significantly from July 2016 to July 2017, with significant increases in the Northeast and among males. Urgent public health action is needed reduce heroin overdoses including increasing the availability of naloxone (an antidote for opioid overdose), linking people at high risk for heroin overdose to medication-assisted treatment, and reducing misuse of opioids by implementing safer opioid prescribing practices. Despite these findings, there are several limitations of these data: not all states sharing data have full participation thus limiting the representativeness of the data; not all ED visits are shared with NSSP; and our case definition may under-identify (e.g., visits missing discharge diagnosis codes and lacking specificity in chief complaint text) or over-identify (e.g., reliance on hospital staff impression and not drug test results) heroin overdose visits. Nonetheless, ED syndromic surveillance data can provide timely insight into emerging regional and national heroin overdose trends.

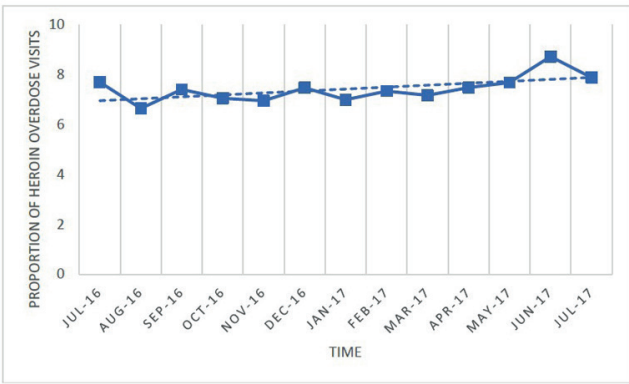

Keywords

Heroin; ESSENCE; Syndromic surveillance

\section{References}

Warner M, Chen LH, Makuc DM, Anderson RN, Minino AM. Drug poisoning deaths in the United States, 1980-2008. NCHS Data Brief 2011(81):1-8.

Rudd RA, Seth P, David F, Scholl L. Increases in Drug and OpioidInvolved Overdose Deaths - United States, 2010-2015. MMWR Morb Mortal Wkly Rep 2016;65(5051):1445-1452.

Spencer MRA, F. Timeliness of Death Certificate Data for Mortality Surveillance and Provisional Estimates. National Center for Health Statistics 2017 
Richards CL, Iademarco MF, Atkinson D, Pinner RW, Yoon P, Mac Kenzie WR, et al. Advances in Public Health Surveillance and Information Dissemination at the Centers for Disease Control and Prevention. Public Health Rep 2017;132(4):403-410.

\section{*Alana M. Vivolo-Kantor}

E-mail: avivolo@cdc.gov 